\title{
The Potpourri of Family Medicine, in Sickness and in Health
}

\author{
Marjorie A. Bowman, MD, MPA, Anne Victoria Neale, PhD, MPH, \\ and Dean A. Seebusen, $M D, M P H$
}

Some aspects of the health of Americans are declining, and family medicine is addressing these ailments. Obesity is one factor, and family physicians provide nutrition counseling. Despite new models such as direct primary care, we have not found an ideal method for providing primary care to everyone in our health system. Not all family physicians report burnout: what is their secret? Scribes: some positives and negatives from one group. Rural America is in need of more family physicians who provide prenatal and natal care, and the proof is in the infant mortality rate. We also have 2 articles on improving pediatric care, and research on the relationship of thrush to mode of infant delivery. We have reviews of new cancer and cholesterol drugs, raising awareness of important knowledge for the primary care clinicians seeing the patients who take these drugs. In addition: tick bites and delayed anaphylaxis-when and how, culture-related skin findings, and helping male perpetrators of domestic violence. (J Am Board Fam Med 2018;31:495-498.)

\section{Morbidity and Obesity}

Some aspects of health in America are declining. ${ }^{1}$ During 2013 to 2014, data from the National Health and Nutrition Examination Survey (NHANES) indicated an overall prevalence of $\geq 2$ comorbidities was $60 \% ; 39 \%$ had $\geq 3$, and $23 \%$ had $\geq 4$ - significantly higher than the prevalence in 1988 (46\% with $>2$ comorbidities). Whites had the greatest increase, and obesity, its causes, and its comorbidities are likely substantial underlying factors. In addition, diagnostic standards keep changing - such as lowering the diagnostic standards for hypertension and diabetes-driving prevalence rates up. Related to increased obesity, Williams et $\mathrm{al}^{2}$ found several important and potentially modifiable predictors of overweight or obese in kindergarten-aged children: birth weight, parental smoking, and infrequent family dinners. These factors increased the risk for children independent of socioeconomic status.

Meanwhile, family physicians in a region of West Virginia with a very high obesity burden noted that patients often lack interest in nutrition counseling. These clinicians indicated they fre-

Conflict of interest: The authors are editors of the $7 A B F M$. quently provide nutrition counseling using common tools, but they do not have much background training. ${ }^{3}$

\section{Health System and Family Medicine}

In a thought piece, Park et $\mathrm{al}^{4}$ conclude that current U.S. payment models do not adequately support the primary care infrastructure known to improve outcomes, that is, team-based community-oriented care that measures the delivery of the functions of primary care.

Some physicians seem to have found their personal key to preventing burnout. In a study of 235 primary care providers practicing in New York City, the rate of provider-reported burnout was $13.5 \%{ }^{5}$-much lower than some studies. For example, Shanafelt et $\mathrm{al}^{6}$ reported a rate of $60 \%$, whereas family physicians taking their American Board of Family Medicine recertification examination had a rate of $25 \% .^{7}$ Perhaps the key is that the New York City providers were all in small independent practices, with small, independent, or their combination being key. About two-thirds were in solo practice.

One type of independent practice is the Direct Primary Care (DPC) model, a newer type of membership medicine in which patients pay a fee for a 
set of family medicine services. Cole ${ }^{8}$ considers DPC against a theoretical model of the 4 attributes of primary care, noting that DPC could have a positive impact on not only the rate of clinician burnout but also the patient-participants' health. However, DPC generally excludes patients who are more likely to be vulnerable and complex, missing one of the important aims for our country's health care system.

Scribes are a potential way to decrease burnout in the face of demands for large increases in documentation. In the study by Zallman et al, ${ }^{9}$ patient volumes were increased after scribes were introduced into a setting with many nonnative Englishspeaking patients. In addition, the proportion of the visit time that was spent face to face increased. Most patients (69\%) felt very comfortable with a scribe in the room. However, a key issue was that the proportion of patients who felt very comfortable with the number of people in the room decreased from $93 \%$ to $66 \%$. Implications of this finding are unknown, but one wonders whether patients do not express some important intimate or concerning thoughts.

\section{Obstetrics and Children's Health}

What is the relation between delivery method and neonatal thrush? This is an unanswered question, with theories based on known differences in the oral microbiome of infants born by cesarean delivery versus vaginal delivery, and known microbiome differences for some other diseases (eg, eczema). Family physician authors analyzed 636 neonate patient records; 127 had a diagnosis of thrush and 509 did not have the diagnosis. ${ }^{10}$ This type of careful research, instigated by questions that arise in the course of patient care, is important.

Powell et $\mathrm{al}^{11}$ took advantage of natural circumstances to review the impact of family physicianprovided obstetric care in rural Alabama. The research was possible because of the loss, then the regain, of family physician obstetric services in a specific area, compared with 2 other areas with continuous service or a lack of family physicianprovided obstetrics service. The authors found that obstetric and/or prenatal services provided by family physicians resulted in an improvement of that county's infant mortality rate. The rate increases and decreases were dramatic (see Figure 2 and Table 3 in that article). This is further evidence that we must, and can, do more to improve obstetric services in rural areas of America and that family physician-provided obstetric care is part of the solution.

A family medicine residency clinic decreased pediatric emergency department utilization through a multiple-prong intervention. ${ }^{12}$ Low-acuity diagnoses of upper respiratory infection decreased by almost half, and visits to the emergency department by pediatric patients with low-acuity diagnoses decreased one-third, from the rates during the same 3 months in the prior year. This led to savings of about $\$ 300,000$ to the Medicaid system. For pediatric well-child care in another academic family medicine office, Wakai et $\mathrm{al}^{13}$ undertook multiple interventions that were associated with significant improvements in before/after comparisons for many items. Despite these improvements, impediments to further gains included parental resistance to venipuncture and specific difficulties for vision and hearing screening.

\section{Clinical Reviews of New Drug Categories and Physician Understanding of Drug Prescribing Materials}

New basic science techniques have led to exciting, innovative medicines for both cancers and cholesterol-related diseases. This issue includes clinical reviews for each category for family physicians. Three types of new cancer medications include checkpoint inhibitors, chimeric antigen receptor (CAR) T cells, and neoantigen therapies. ${ }^{14}$ Checkpoint inhibitors are becoming very common. Of note for family physicians, these inhibitors may lead to autoimmune-related side effects such as pneumonitis, hypothyroidism, and colitis. These side effects may present at any time, from immediately to several months after beginning therapy, so family physicians need to be alert. CAR T cells are $T$ cells that undergo genetic engineering to express a particular antigen receptor related to the cancer. They are powerful but have substantial side effects, in particular what is known as cytokine release syndrome, which affects about two thirds of patients. Personalized vaccines related to tumor-associated antigens are now available but uncommonly used.

In 2015, the Food and Drug Administration approved a new family of lipid-lowering agents known as proprotein convertase subtilisin/kexin 
type 9 inhibitors; these are biologically active molecules that have a different mechanism of action than statins. They decrease serum low-density lipoprotein cholesterol compared with statin therapy alone, ${ }^{15}$ but they are expensive and require subcutaneous injections.

Just as there are new medications, there continues to be new statistical methods and terms compared with what the average physicians learned in medical school. For example, physicians demonstrated low to moderate knowledge about clinical trial-related terms found in promotional prescription drug materials. ${ }^{16}$ Less than one fourth of the clinician-participants could provide an accurate description of several terms, including per-protocol analysis, modified intent to treat, adjusted mean, and noninferiority margin, and poor understanding of several others. This could lead to significant misunderstandings of the true usefulness of the related medications. Rather than a simple "teach physicians more statistics," we advocate improving prescription drug materials for better readability and understanding.

\section{Clinical Medicine}

This issue also contains several other clinical articles. Perhaps the most fascinating is on $\alpha$-gal allergy. ${ }^{17}$ Talk about roundabout: someone gets bitten by a tick, then experiences anaphylaxis hours after visiting a buffet restaurant. What would be your guess as to the cause of the allergic reaction? Several reports have now been published of Lone Star tick bites (which have $\alpha$-gal in their saliva) related to the development of mammalian meat allergy, which in turn causes anaphylaxis that may be delayed. The authors add another patient case to the growing list of those reported to be affected. Mammalian meat $\alpha$-gal is in many foods (eg, gelatins), and sometimes the allergy extends to milk products. It can be difficult for patients to avoid all exposure; thus epinephrine pens should be provided.

Einterz ${ }^{18}$ provides a helpful review of scarification and burning of the skin, excision of body parts, and subcutaneous insertion of foreign material that are common to certain cultures. In a separate article, authors from the Centers for Disease Control and Prevention provide the sad news that the majority of men over age 70 who had prostate screening antigen (PSA) testing in the past year reported that their physicians suggested and ordered the test, suggesting a lack of adequate shared decision making. ${ }^{19}$ The US Preventive Task Force recommends against PSA testing and for shared decision making if doing so. We hope new, improved testing methods can move us away from such automatic PSA testing. van Dipten et $\mathrm{al}^{20}$ found that patients had many misconceptions about kidney disease; perhaps the most important is that they seemed unaware of the relation of kidney disease to lifestyle and cardiovascular disease. Read the article for more specific details.

Perhaps not surprisingly, Sherman et $\mathrm{al}^{21}$ found that patients taking continuous opioid therapy typically reported favorable perceptions of doctor-patient trust in managing opioid pain medicines. However, implementing opioid risk-reduction initiatives may lead to reduced levels of trust for a minority of these patients. This suggests that it may be possible to implement opioid risk-reduction initiatives while sustaining high levels of doctor-patient trust for most, but not all, patients receiving chronic opioid therapy. Meanwhile, patient satisfaction is still considered a major measure of physician quality.

Penti et $\mathrm{al}^{22}$ provide a review of working with male perpetrators of domestic abuse. In addition to many valuable recommendations, the authors commend referrals to a batterer intervention program. If no batterer intervention program is locally available, or if the patient is unwilling to attend one, a physician should refer the abuser to a therapist who is trained to work with perpetrators of interpersonal violence.

To see this article online, please go to: http://jabfm.org/content/ 31/4/495.full.

\section{References}

1. King DE, Xiang J, Pilkerton CS. Multi-morbidity trends in United States adults, 1988-2014. J Am Board Fam Med 2018;31:503-13.

2. Williams AS, Ge B, Petroski G, Kruse RL, McElroy JA, Koopman RJ. Socioeconomic status and other factors associated with childhood obesity. J Am Board Fam Med 2018;31:514-21.

3. Nair D, Hart A. Family physicians' perspectives on their weight loss nutrition counseling in a high obesity prevalence area. J Am Board Fam Med 2018;31: 522-28.

4. Park B, Gold SB, Bazemore A, Liaw W. How evolving United States payment models influence primary 
care and its impact on the quadruple aim. J Am Board Fam Med 2018;31:588-604.

5. Blechter B, Jiang N, Cleland C, Berry C, Ogedegbe $\mathrm{O}$, Shelley D. Correlates of burnout in small independent primary care practices in an urban setting. J Am Board Fam Med 2018;31:529-36.

6. Shanafelt TD, Hasan O, Dyrbye LN, et al. Changes in burnout and satisfaction with work-life balance in physicians and the general US working population between 2011 and 2014. Mayo Clin Proc 2015;90: 1600-13.

7. Puffer J, Knight HC, O’Neill TR, et al. Prevalence of burnout in board certified family physicians. J Am Board Fam Med 2018;30:125-6.

8. Cole ES. Direct primary care: applying theory to potential changes in delivery and outcomes. J Am Board Fam Med 2018;31:605-11.

9. Zallman L, Finnegan K, Roll D, Todaro M, Oneiz $\mathrm{R}$, Sayah A. Impact of medical scribes in primary care on productivity, face-to-face time and patient comfort. J Am Board Fam Med 2018;31:612-19.

10. St. Clair-Brown TT, Schwerer KE, Dogbey GY. Neonatal thrush is not associated with mode of delivery. J Am Board Fam Med 2018;31:537-41.

11. Powell J, Skinner C, Lavender D, Avery D, Leeper J. Obstetric care by family physicians and infant mortality in rural Alabama. J Am Board Fam Med 2018; 31:542-49.

12. Davis T, Meyer A, Beste J, Batish S. Decreasing low acuity pediatric emergency room visits with increased clinic access and improved parent education. J Am Board Fam Med 2018;31:550-57.

13. Wakai T, Simasek M, Nakagawa U, Saijo M, Fetters MD. Screenings during well-child visits in primary care: a quality improvement study. J Am Board Fam Med 2018;31:558-69.
14. Sriratana P, Norton J. New immunotherapies in oncology treatment and their side effect profiles. J Am Board Fam Med 2018;31:620-27.

15. Shahreyar M, Salem SA, Nayyar M, George LK, Garg N, Koshy SKG. Hyperlipidemia: management with proprotein convertase subtilisin/kexin type 9 (PCSK9) inhibitors. J Am Board Fam Med 2018;31: 628-34.

16. Moynihan CK, Burke PA, Evans SA, O’Donoghue AC, Sullivan HW. Physicians' understanding of clinical trial data in professional prescription drug promotion. J Am Board Fam Med 2018;31:645-49.

17. Kaplan AC, Carson MP. Diagnosing meat allergy after tick bite without delay. J Am Board Fam Med 2018;31:650-52.

18. Einterz E. Recognizing culture related findings on refugee physical examinations. J Am Board Fam Med 2018;31:653-57.

19. Li J, Ding H, Richards TB, Martin I, Kobrin S, Marcus PM. Prostate-specific antigen testing initiation and shared decision-making: Findings from the 2000 and 2015 National Health Interview Surveys. J Am Board Fam Med 2018;31:658-62.

20. van Dipten C, Grauw WJC, de Wetzels JFM, Assendelft WJJ, Scherpbier-de Haan ND, Dees MK. What patients with mild to moderate kidney disease know, think, and feel about their disease: an in-depth interview study. J Am Board Fam Med 2018;31:57077.

21. Sherman KJ, Walker RL, Saunders K, et al. Doctorpatient trust among chronic pain patients on chronic opioid therapy after opioid risk reduction initiatives: a survey. J Am Board Fam Med 2018;31:578-87.

22. Penti B, Timmons J, Adams D. The role of the physician when a patient discloses intimate partner violence perpetration: a literature review. J Am Board Fam Med 2018;31:635-44. 